\title{
Amplitude Characteristics Low-frequency Compensation
}

\author{
Yinghui Xie ${ }^{1, a^{*}}$ \\ ${ }^{1}$ Hunan Software Vocational College, Xiangtan, 411100, China \\ matlab_wjf@126.com
}

Keywords: Sensor; Vibration; Amplitude-frequency characteristics; Compensating circuit; Transfer function; Sensitivity

\begin{abstract}
The hydropower generating units vibration parameter is an important indicator to monitor its roll-stabilization. To measure the low-frequency vibration of the large and middle scale of hydropower generating units, according to frequency characteristic compensation principle of the vibration sensor, a low-frequency compensating circuit was designed to extend the frequency characteristic to the low frequency region. The mathematical model of the magnetoelectric dromometer vibration sensor and the design of the compensation circuit were detailed. The amplitude-frequency characteristics of the sensor and its mathematical model were comparatively analyzed before and after compensation respectively. The experimental result shows that the design could extend the amplitude-frequency characteristics to the low frequency region, and have good sensitivity and linearity.
\end{abstract}

\section{Introduction}

Hydro accident showed vibration fault [1]. Medium-sized hydro group transfer frequency is low, about $1 \sim 2 \mathrm{~Hz}$, and unit water vortex-induced vibration which hydro draft Tube produces is lower, about $1 / 5$ to $1 / 3$ of rotation frequency. In addition, once the accident occurred during hydropower units operation, which load shedding and load rejection transition process, vibration signal frequency will be lower [2]. In magnetic vibration velocity sensors, smaller natural frequency, the greater the volume. Considering the ease of installation of the sensor, the magnetic vibration velocity sensor is widely used in engineering, and its natural frequency limit of is about $2.5 \mathrm{~Hz}$, but also the measurement frequency is higher and 2 to 3 times than the natural frequency of vibration sensor[3]. If the sensor is without compensation, and the direct the measurement is operated, the measurement is not accurate. How to design compensation circuit, and the vibration sens frequency characteristics is allowed to expand the low-frequency or to meet the requirements of the low-frequency test, it is the focus of the study.

\section{Establish and Validate of the Vibration Sensor Model}

Operating Principle of the Oscillation Sensor: Magnetic vibration velocity sensors have been widely applied in low-frequency sensor ,because of these advantages whch their output signal is large, the follow-up circuit is simple, anti-jamming capability is strong. As shown in Figure 1, the vibration sensor is fixed with a permanent magnet inside, and in the outer casing, the magnetic circuit of the magnet is enclosed within the housing. Two circular spring upper and lower is fixed between the magnet and the housing. they support the coil, and are surrounded by the coil with mass $m$ and without touching the magnet. When the external action of the vibration sensor, the relative motion is made betwen the magnet and the coil, the coil is cutting magnetic induction line, and is generating a voltage signal. 


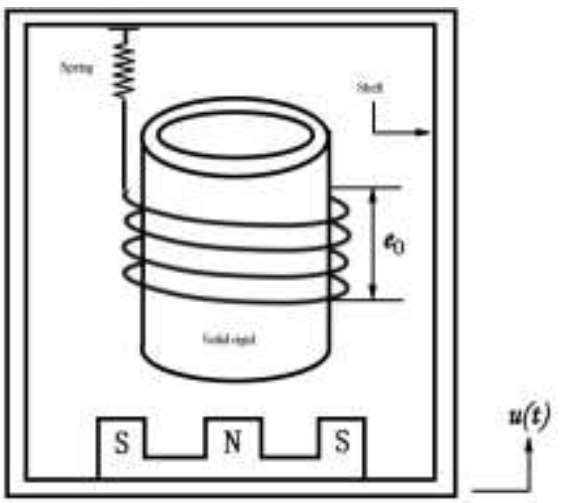

Figure 1. Magnetic vibration sensor structure diagram

Model building : It is an inertial sensor, the mechanical model can be simplified as a single system with the degree of freedom, which is composed of three-part system of a spring with elastic coefficient $\mathrm{k}$, inertial mass $\mathrm{m}$ and damping $\mathrm{C}[4]$. As shown in Figure 2.

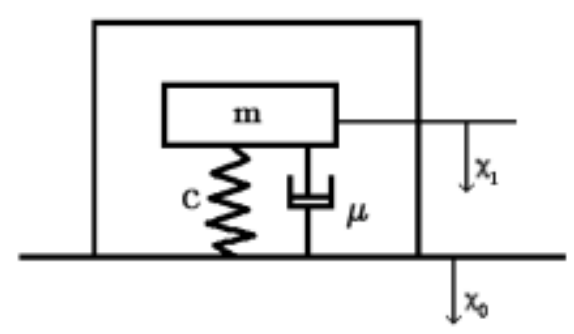

Figure 2. Mathematical Model

The motion equation is

$$
m x_{1}^{\prime \prime}+\mu\left(x_{1}^{\prime}-x_{0}^{\prime}\right)+c\left(x_{1}-x_{0}\right)=F(t)
$$

Which : $\mathrm{m}$ - inertial body mass (coil)

$\mathrm{C}$ - spring stiffness

$\mu$ - Damping

$x_{0}$ - The absolute displacement of the base housing

$x_{1}$ - The absolute displacement of the inertial body

$x_{0}^{\prime}$ - The speed of the base housing

$x_{1}$ - The speed of the inertia body

$x_{1}^{\prime \prime}$ - Acceleration of the inertial body

$F(t)$ - The forces acting on the inertial body

Take $F(t)=0$;

$\omega_{0}^{2}=c / m, \omega_{0}$ is characteristic angular frequency

$2 \xi_{0} \omega_{0}=\mu / m, \xi_{0}$ is damping ratio;

$x_{r}=x_{1}-x_{0}$, Mass displacement is relative to vibration sensor housing. (1) by the Laplace transform as follows: 


$$
H(s)=\frac{X_{r}(s)}{X_{0}(s)}=\frac{s^{2}}{s^{2}+2 \varsigma_{0} \omega_{0} s+\omega_{0}^{2}}
$$

Vibration sensors coil is are cutting magnetic inductionline, the output voltage is:

$$
u_{0}=B l x_{r}^{\prime}
$$

By Laplace transform as follows:

$$
U_{0}(s)=B l s X_{r}(s)=k_{0} s X_{r}(s)
$$

Which

B - magnetic field strength

$l$ - the length of the coil which is cutting magnetic field lines

$k_{0}$ - Sensor sensitivity coefficient

By the formula (2), (4), a vibration sensor output response:

$$
\begin{aligned}
U_{0}(s) & =k_{0} H(s) s X_{0}(s) \\
& =\frac{-k_{0} s^{2}}{s^{2}+2 \xi_{0} \omega_{0} s+\omega_{0}^{2}} s X_{0}(s)
\end{aligned}
$$

Vibration sensor transfer function is:

$$
G_{1}(s)=\frac{-k_{0} s^{2}}{s^{2}+2 \xi_{0} \omega_{0} s+\omega_{0}^{2}}
$$

In CDJ-Z2.5C vibration sensor as a reference, to establish the mathematical model. Natural frequency $f_{0}=2.5 \pm 10 \% \mathrm{~Hz}$, take value 2.5. Damping is $\xi_{0}=0.7 \pm 10 \%$, take value 0.75 . $\omega_{0}^{2}=\left(2 \pi f_{0}\right)^{2}=246.74,2 \xi_{0} \omega_{0}=23.56$. If $k_{0}=1800$, the formula (6) can be written as:

$$
G_{1}(s)=\frac{-1800 s^{2}}{s^{2}+23.56 s+246.74}
$$

Matlab simulating curve with the amplitude-frequency characteristic , as shown in Figure 3, curve B is the simulation results. 


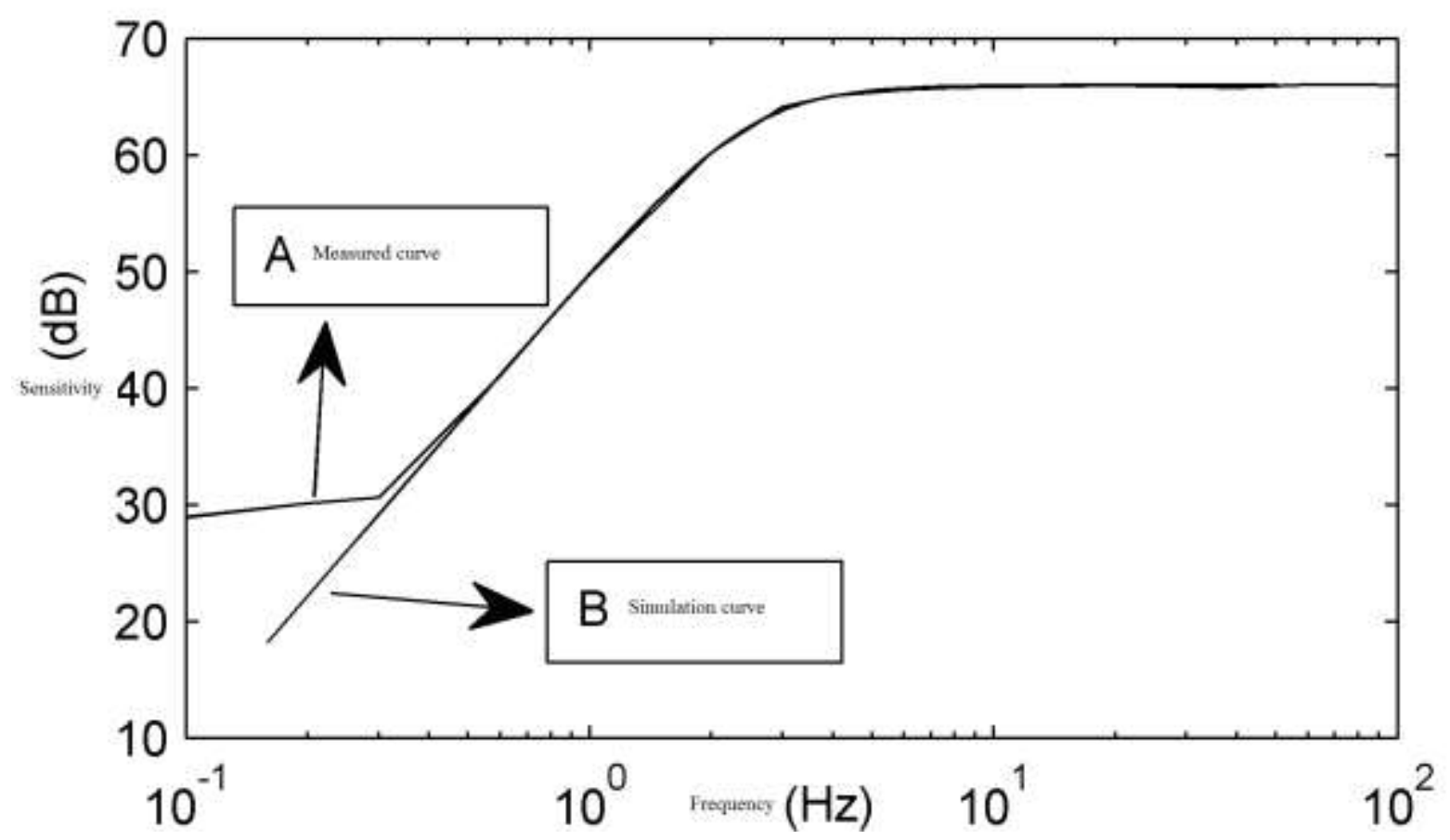

Figure 3. Amplitude-frequency characteristic curve

Verify Model: Equipment used in the experiment: one CDJ-Z2.5C vibration sensor, an integrated vibration tester. The vibration sensor is put vertically on the vibration generator, the output of the sensor is directly connected to the tester's input. Set the output vibration level, vibration level is $0.5 \mathrm{~cm}$ / s.

Note: Sensitivity $=$ voltage $/$ vibration level, the unit is $\mathrm{mV} / \mathrm{cm} \cdot \mathrm{s}^{-1}$.

In Table 1, the sensitivity is the voltage whic is normalized by dividing the vibration level, unit is $\mathrm{mV} / \mathrm{cm} \cdot \mathrm{s}^{-1}$. The measured amplitude-frequency characteristic curve is curve A which is shown in Figure 3. Comparison of the curves A, B in Figure 3, it can be seen that the simulation and measured curve are same when frequency is larger than $0.3 \mathrm{~Hz}$, almost exactly. The vibration frequency is less than $0.3 \mathrm{~Hz}$, output of the sensor itself is small, and because of the floor and all noise, the relative output is high. The lower the frequency, the larger the error. Comprehensive assessment is that model is feasible.

\section{Design of Compensation Aspects}

Compensation Principle : In order to extend the frequency response of the low-frequency magnetic vibration velocity sensors, the circuit compensation method is required. There ae two compensation forms in the circuit compensates, which are feedback compensation and series compensation, and the feedback compensation is with lower natural frequency, while the damping ratio is reduced, stability is poor, but also it is easy to produce oscillations [5]. It is used in series compensation. The sensor output voltage is in series with a compensation network $\mathrm{C}(\mathrm{s})$, so that the pole of the original transfer function G1 (s) is eliminated by the C (s) zero point, C (s)'s pole becomes the pole of the transfer function $\mathrm{G}$ (s) after passing compensation, so the low-frequency output characteristics of the sensor can been changed by changing the compensation network's poles. The transfer function of the compensation aspects were given in Literature [5-8], equation (8) below, this transfer function can be decomposed by three parallel filtering part, namely all-pass, low-pass, band-pass filtering links .

$$
\begin{aligned}
C(s) & =\frac{s^{2}+2 \xi_{0} \omega_{0} s+\omega_{0}^{2}}{s^{2}+2 \xi_{1} \omega_{1} s+\omega_{1}^{2}} \\
& =1+\frac{\omega_{0}^{2}-\omega_{1}^{2}}{s^{2}+2 \xi_{1} \omega_{1} s+\omega_{1}^{2}}+\frac{2\left(\xi_{0} \omega_{0}-\xi_{1} \omega_{1}\right) s}{s^{2}+2 \xi_{1} \omega_{1} s+\omega_{1}^{2}}
\end{aligned}
$$


Where $\xi_{1}$ is the damping ratio of compensation aspects, which reflects the frequency characteristic in the oscillation links, and does not directly reflect the frequency response of the filter circuit. So in this paper, the damping ratio $\xi_{1}$ is replaced by the equivalent quality factor $\mathrm{Q}, \mathrm{Q}$ is a parameter which reflects the frequency response of the filter circuit. As shown in formula (9):

$$
C(s)=\frac{s^{2}+2 \xi_{0} \omega_{0} s+\omega_{0}^{2}}{s^{2}+\frac{\omega_{1}}{Q} s+\omega_{1}^{2}}
$$

Where $\omega_{1}=2 \pi f_{1}$ is characteristic angular frequency which is compensated. After the compensation, transfer function $\mathrm{G}$ (s) of the vibration sensor is:

$$
\begin{aligned}
G(s) & =G_{1}(s) \bullet C(s) \\
& =\frac{-k_{0} s^{2}}{s^{2}+\frac{\omega_{1}}{Q} s+\omega_{1}^{2}}
\end{aligned}
$$

Compensation circuit $\mathrm{C}(\mathrm{s})$ is designed, so that $\omega_{1}<\omega_{0}$, and $\mathrm{Q}$ is the best equivalent quality factor. Compensated system maintains the original vibration sensor high mechanical properties unchanged, and the natural frequency depends entirely on the compensation circuit which is connected in series, thus in compensated sensor system, the original sensor volume is small, and performance characteristics is good, but also low-frequency output characteristics is improved .

Vibration sensor compensation circuit design: To meet the measurement requirements of hydroelectric generating low-frequency vibration, the natural frequency of the sensor is needed to expand from $2.5 \mathrm{~Hz}$ to the low-frequency $0.2 \mathrm{~Hz}$, to maintain the best quality factor. That is $f_{1}=0.2 \mathrm{~Hz}$, $\mathrm{Q}=0.7$. The compensation link $\mathrm{C}(\mathrm{s})$ is divided into:

$$
C(s)=1+\frac{k_{1} A_{V F} \omega_{1}^{2}}{s^{2}+\frac{\omega_{1}}{Q} s+\omega_{1}^{2}}+\frac{k_{2} A_{V F} \omega_{1} s}{s^{2}+\frac{\omega_{1}}{Q} s+\omega_{1}^{2}}
$$

In the formula,.

$$
k_{1}=\frac{\omega_{0}^{2}-\omega_{1}^{2}}{\omega_{1}^{2} A_{V F}}, k_{2}=\frac{2 \xi_{0} \omega_{0}-\omega_{1} / Q}{\omega_{1} A_{V F}}
$$

Among them, ${ }_{V F}$ for the same phase scaling circuit voltage gain.

By formula (11), it can be seen that the compensation aspect is the parallel together with all-pass, low-pass, band-pass three aspects, the structure is shown in Figure 4. 


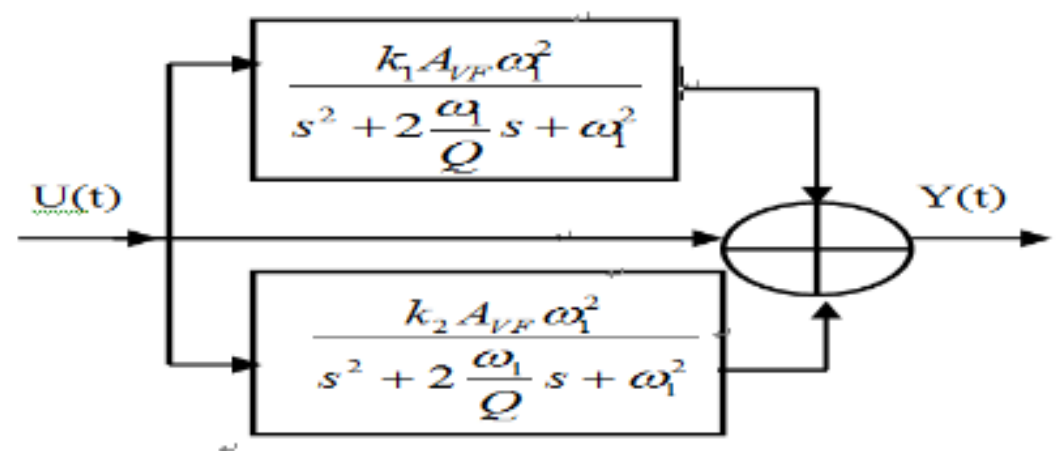

Figure 4. Compensation link Structure Figure

The ratio between these three gain is adjusted, zerosof the compensation link and poles of the original system are made offset, allowing the system to achieve the desired frequency characteristics. Because of the integrated operational amplifier module features itself, little DC offset will been produced, after amplification, this can not be ignored, so after the filter circuit, the DC offset is removed in series with a first-order high-pass filter circuit . The cutoff frequency should be much less than $0.1 \mathrm{~Hz}$. Figure 5 is a compensation circuit wiring diagram.

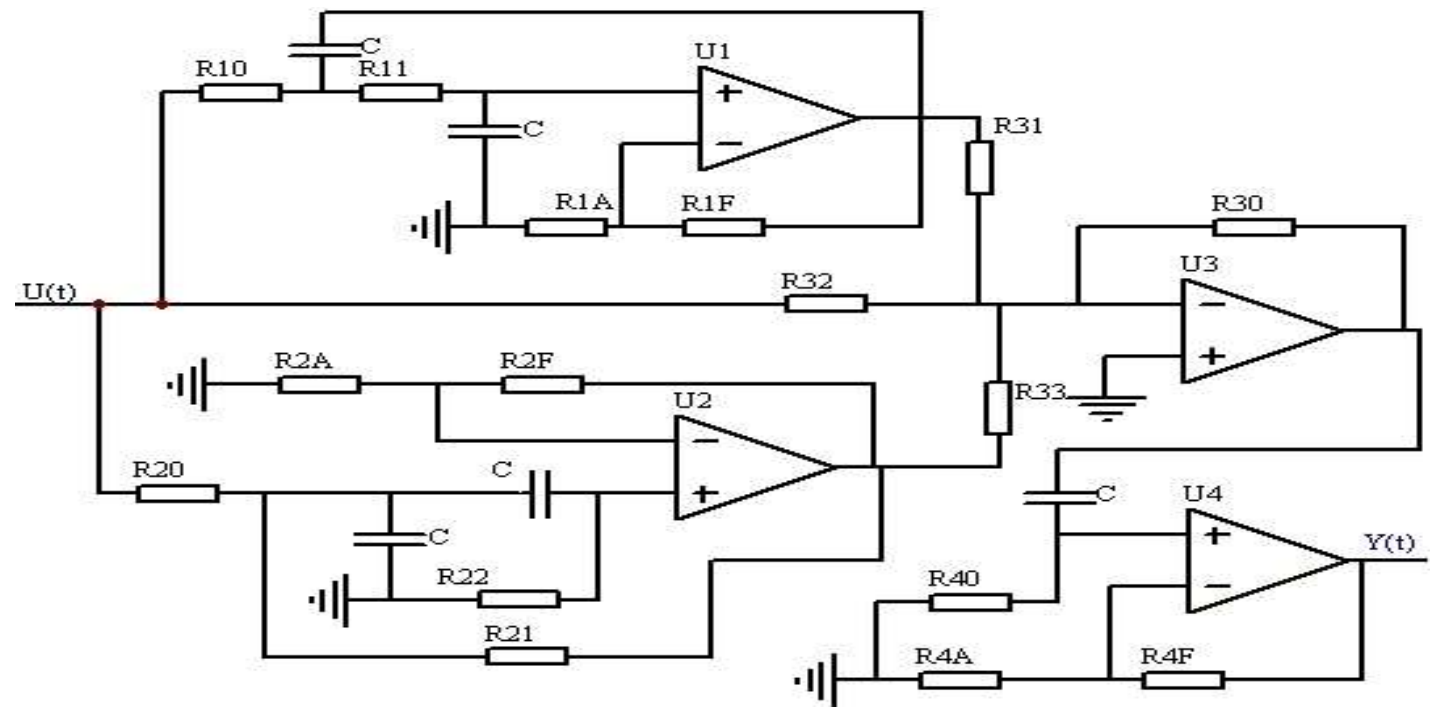

Figure 5. Compensation circuit wiring diagram

Wherein: R10、R11、R1A、R1F、C、U1 constitutes a second-order low-pass filter circuit; R20、R21、R22、R2A、R2F、C、U2 constitutes a second order band-pass filter circuit; R32 is an all-pass circuit; R30、R31、R32、R33、U3 constitute an addition circuit; R40、R4A、R4F、C、 $\mathrm{U} 4$ form a first order high pass filter. To reduce system circuit noise, each resistor in the circuit should be trillion level and below, so the capacitance value $\mathrm{C}=10 \mathrm{uF}$. to simplify the calculation, so that $\mathrm{R} 10=\mathrm{R} 11=\mathrm{R} 20=\mathrm{R} 22=\mathrm{R} ; \mathrm{R} 22=2 \mathrm{R} ; \mathrm{R} 30=\mathrm{R} 32=100 \mathrm{k} \Omega$, The design requirements was: $\mathrm{R}_{1 \mathrm{~A}}=\mathrm{R}_{2 \mathrm{~A}}=\mathrm{R}_{\mathrm{A}} ; \mathrm{R}_{1 \mathrm{~F}}=\mathrm{R}_{2 \mathrm{~F}}=\mathrm{R}_{\mathrm{F}}$. And $V_{A F}=1+\frac{R_{F}}{R_{A}}=\frac{3 Q-1}{Q}, \mathrm{R}_{\mathrm{A}} \| \mathrm{R}_{\mathrm{F}}=2 \mathrm{R}, \omega=2 \pi f=1 / R C, \mathrm{R}_{31}=\mathrm{R}_{30} / \mathrm{k}_{1}, \mathrm{R}_{33}$ $=\mathrm{R} 30 / \mathrm{k} 2$, so the parameter values of the circuit is about $\mathrm{R}=80 \mathrm{k} \Omega, \mathrm{RA}=440 \mathrm{k} \Omega, \mathrm{RF}=250 \mathrm{~K} \Omega, \mathrm{R} 31=$ $1 \mathrm{k} \Omega, \mathrm{R} 33=9 \mathrm{k} \Omega$. For a first-order high-pass filter, parameter is not strictly required to achieve the blocking effect. Due to the low signal to noise ratio of low frequency sensor output voltage, low-frequency amplitude of the original system is bigger than the theoretical value. R20, R21, R22 resistance can be increased, so that the amplitude-frequency characteristic of the band rejection filter circuit is translated to the high frequency. In order to increase the output voltage of the sensor system, the voltage drop of the circuit is compensated. 


\section{Analysis}

Development of Low-frequency : The curves A, B of Figure 6 is respectively the amplitude-frequency characteristic curve before and after the system compensation[9-11]. And generally, the-3dB frequency corresponding about $70 \%$ of the flat area is the natural frequency of the system. For example, the compensation is not increased in circuit, if frequency is equal to $20 \mathrm{~Hz}$, the sensitivity is 1993 , and its $70 \%$ is 1395 . From the data in the table, the natural frequencies are showed between $2-3 \mathrm{~Hz}$, about $2.5 \mathrm{~Hz}$, and it is the actual match. After adding the compensation circuit, if frequency is equal to $20 \mathrm{~Hz}$, the sensitivity is 1945 and its $70 \%$ is 1361 , its natural frequency should be between $0.1-0.2 \mathrm{~Hz}$ which is seen from Table 2, approximately $0.18 \mathrm{~Hz}$. It can be seen in Figure 6 that curve A natural frequency is about $2.5 \mathrm{~Hz}$, the natural frequency of curve $\mathrm{B}$ is about $0.18 \mathrm{~Hz}$, thus the system is expanded to 13.8 times of low frequency, that is $0.18 \mathrm{~Hz}$, the design requirements are meet.

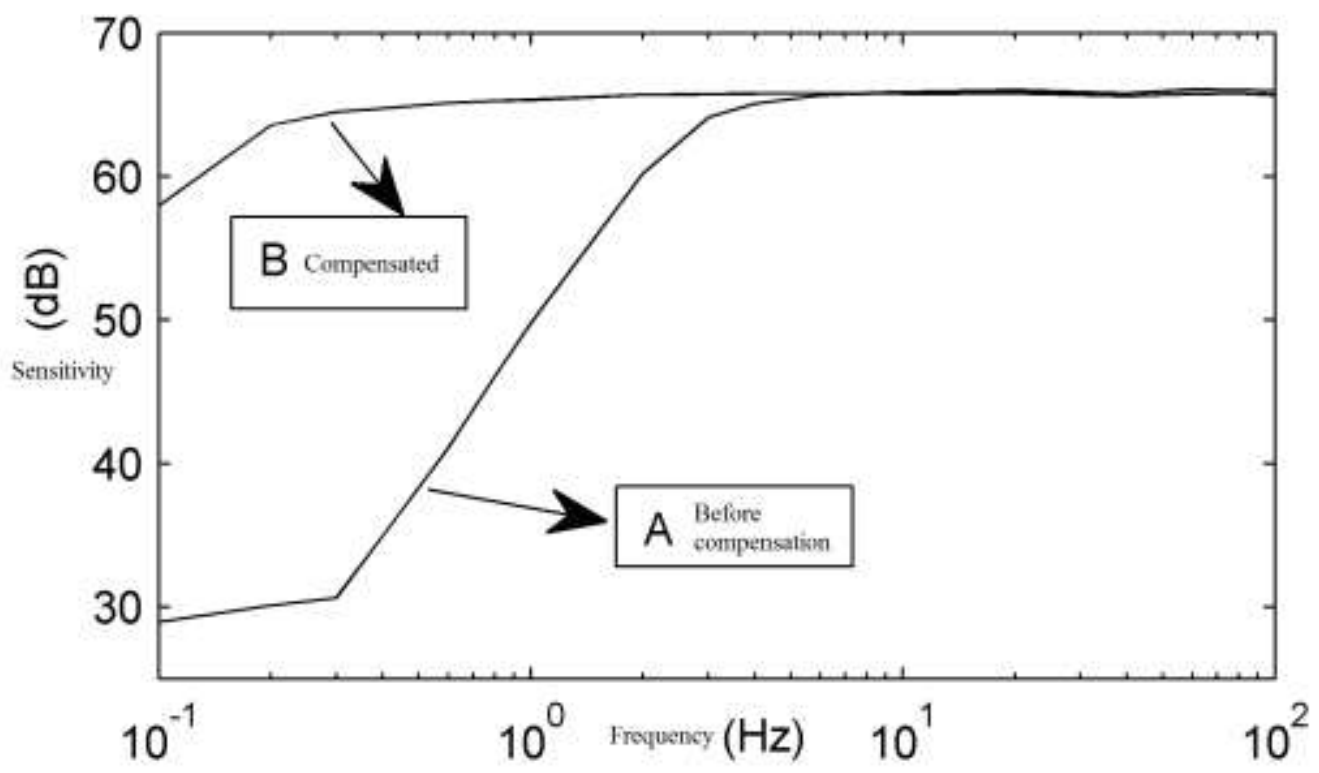

Figure 6. Compensated amplitude-frequency characteristic curve

Linearity: Sensitivity in $20 \mathrm{~Hz}$ is taken as standard before and after compensation, the linearity relative error of the each frequency is calibrated. In $80 \mathrm{~Hz}$ or less, the linearity relative error of each frequency after compensation system is relatively much smaller than one of the previous compensation, the compensated relative error at the frequency $100 \mathrm{~Hz}$ was only $1.2 \%$, so the compensating linearity has greatly improved than one before compensation.

Sensitivity: Vibration data $0.5 \mathrm{~cm} / \mathrm{s}$ is for a standard vibration level, the relative error of the sensitivity is calculated by a vibration data acquisition another $0.8 \mathrm{~cm} / \mathrm{s}$ vibration level, so the sensitivity stability of the compensated system is the assessed. The relative error is 0.005 or less in the sensitivity, a sensitivity of the system remains stable after compensation.

\section{Conclusion and Outlook}

In the design of the compensation aspects, the equivalent quality factor is cited by Q, so that the compensation circuit design of the magnetic vibration velocity sensor is simple and straight victory. The low-frequency system is developed to low-frequency after compensated, there are sensitivity, linearity, more comprehensive analysis, these are indicating that the sensor system performance is good after compensating, the design of the compensation aspects is proved reasonably practicable.

\section{References}

[1] SHEN Dong, CHU Fu-tao, CHEN Si. Diagnosis and Identification of Vibration Accident for 
Hydrogenerator Unit[J], JOURNAL OF HYDRODYNAMICS ,2000,15（1）:129-133.

[2] ZHENG Jinju, Yu Shuibao, Sun Xiaoqin, A New Magnetic Sensor[J], CHINESE JOURNAL OF SCIENTIFIC INSTRUMENT ,2005, 26(8) :40-44.

[3] XIAO Mingwei, YANG Yongming, ZHONG Yulin. Research on the measurement of low-frequency vibration of water turbine generators[J], TRANSDUCER AND MICROSYSTEM TECHNOLOGIES, 2011, 30(5)

[4] Liu yu. The Establishment of Vibration Sensor Verification Device[J], Metrology \& Measurement Technique, 2012, 39(11)

[5] Yunxiao Fan, Hua Liu, Nong Wang. Study on Ultra-Low Frequency Vibration Sensor by Using Magnetoelectric Speedometer[J], JOURNAL OF UNIVERSITY OF SCIENCE AND TECHNOLOGY, 2001,20(3):41-43

[6] LI Ming-guo, SUN Rong-xiang, LI Yong. Research on Vibration Sensor of Ultra Low Frequency[J], COLLIERY MECHANICAL \& ELECTRICAL TECHNOLOGY ,2008,(3):37-39

[7] Along yu, Weiyi Huag. Research on Methods of Improvement for Amplitude/Frequency Characteristic of Vibration Velocity Transducer[J], PROCESS AUTOMATION INSTRUMENTATION , 2004,3(4):289-291,295.

[8] Yu Shuibao, Study on optimizing technique for transfer function of the ULF absolute vibration sensor[J], CHINESE JOURNAL OF SCIENTIFIC INSTRUMENT ,2006, 27(8):940-942.

[9] Yang X S. Low-frequency characteristic extension technique research for velocity vibration sensor[J].Earthquake Engineering and Engineering Vibration,2004,(02):139-146.

[10] A1Bedoor B O. Blade Vibration Measurement in Turbo-machinery:Current Status[J].Shock and Vibration Digest,2002,(06):455-461.

[11] Wang Chao,Tang Huang,Xiao Qian. The Application of Wide Spectrum Laser in Noncontact Vibration Measurement[J].Microwave and Optical Technology Letters,2009,(12):2858-2861. 